\title{
COUPLING AND ASSEMBLY ELEMENTS USING MICROFABRICATION TECHNOLOGIES
}

\author{
Marius Popa ${ }^{1}$, Cristinel Ilie ${ }^{2}$, Daniel Lipcinski ${ }^{3}$, Ionel Chirita ${ }^{4}$, \\ Nicolae Tanase ${ }^{5}$, Simona Apostol ${ }^{6}$ \\ ${ }^{1}$ National Institute for Research and Development in Electrical Engineering ICPE-CA \\ Splaiul Unirii 313, 030138, sector 3, Bucharest \\ marius.popa@icpe-ca.ro ,cristinel.ilie@icpe-ca.ro, daniel.lipcinski@icpe-ca.ro, \\ ionel.chirita@icpe-ca.ro, nicolae.tanase@icpe-ca.ro, simona.apostol@icpe-ca.ro
}

\begin{abstract}
The purpose of this paper is to presents the conception, achievements, measurement and data analysis regarding the connecting and the assembling of the components of a MEMS electromagnetic microactuator, cantilever type.

The microactuator is composed of a fixed lower part on which was processed a flat coil and a mobile upper part that contains a cantilever and a permanent magnet or an array of permanent magnets.

The two parts must be coupled and joined together.

The purpose of the study is to realize the high precise coupling and fixing parts, to ensure a precisely relative positioning and high repeatability between parts position, using a simple and low cost technology.

There were processed and analyzed six constructive shapes, male-female type, each one of them in two dimension types.

The structures were made by UV laser photolithography, using SU8 3050 resist.

The measurements were made using optical microscopy and white -light interferometry (optical profilometry).

Dimensional measurements were performed for the coupled parts male-female type, also, the surface roughness was measured and the thickness uniformity of the structures.

The conclusions establish the best choice of the constructive shapes for the connecting elements, according to a particular application.
\end{abstract}

Keywords: Microactuator, Cantilever, UV Laser Photolithography, SU8 3050 Resist, Experimental Validation, White -Light Interferometry Measurement.

\section{Introduction}

The magnetic MEMS present a new class of micro devices with great potential and applications. Using the same technology as for conventional MEMS and incorporating magnetic materials as the sensing or actuating element, they offer new capabilities and open new markets within the information technology, automotive, biomedical, space and instrumentation. Magnetic MEMS are based on electromagnetic or magnetic interactions between magnetic materials and active electromagnetic coils or passive magnetic field sources such as permanent magnets. Magnetic materials can be deposited on micro parts, and assembled in micro devices [1].

In addition, magnetic MEMS devices with a permanent magnet have benefits from constant energy stored in the hard magnetic materials, which include low power consumption, favorable scaling and simple electronic circuitry, compared to a variable reluctance-type electromagnetic actuator. The limited number of electromagnetic actuators in MEMS is partially due to the difficulties in fabrication of micro-sized electromagnetic actuators with silicon-based MEMS technology, and partially because of some misconceptions about electromagnetic actuation.

Generally, magnetic MEMS devices are known to have several advantages over electrostatic MEMS devices. These advantages include generation of long-range force and deflection with low driving voltage, even under harsh environment [2]. Furthermore, permanent magnets can achieve relatively high energy density in microstructures, compared to other energy storage devices in the micro scale. They can also be controlled with low cost microcontrollers and low voltage [3-6].

The development of the electromagnetic micro actuators was stimulated by the development of the LIGA technology and the SU8 photoresist family, 
which allowed the development of MEMS applications with thick layers up to the order of hundreds of micrometers. This technology involves several operations described below.

\section{Technical Solution}

The basically sketch of the chosen technical solution is presented in fig. 1.

It is noted that in both cases the actuator is composed of an array of permanent magnets (2)

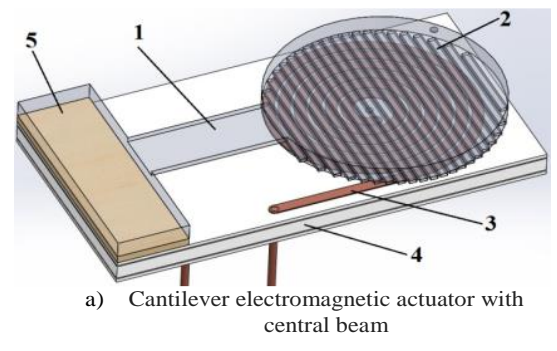

fixed to the cantilever (1), a plane coil (3) made on a rigid support (4) and a coupling element (5).

The coupling element connects the movable element (cantilever with permanent magnets) and the fixed system (planar coil).

The alignment of the magnetic fields of the two actuating elements, the permanent magnets (2) and the planar coil (3), represents an essential step in obtaining of a functional performance features [7].

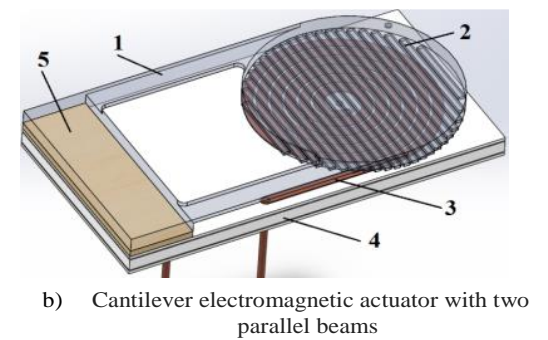

Fig. 1. Cantilever type electromagnetic actuators

\section{Working Principle}

The system of forces participating in the equilibrium, as outlined in figure 1 , is given by the electromagnetic interaction force between the coil and the permanent magnet array and the mechanical force of the cantilever.

$$
F_{e}=F_{e m}
$$

Where $F_{e}$ represents elastic force and $F_{e m}$ represents electromagnetic force.

The control of the tilting angle is achieved by controlling the magnetic interaction between the permanent magnet, which together with the cantilever (1), forms the movable system and the magnetic field of the coil fixed to the fixed support (4). The control is made by controlling the coil current.
The assembly between the two fixed and mobile systems is made by means of the connecting piece (5), provided with coupling and assembly elements, manufactured on a micrometric scale.

\section{Connection Elements}

A good operation, in terms of positioning and repeatability can be achieved only using coupling (connecting) elements as (more) precise and easier to manufacture.

They have analyzed several couplingconnection elements, and it will determine the most suitable for our application form (in accordance with the technological capabilities we have) and accuracy (with the appropriate tolerances). The analyzed forms come from the technical literature [8], in conjunction with the collective experience and are presented in Figure 2.

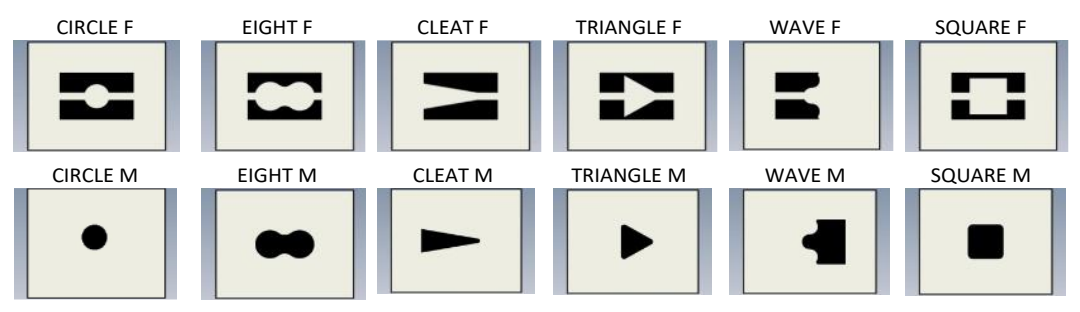

Fig. 2. The drawings of test structures for coupling elements

The rectangle dimensions on which the coupling structures was manufactured was $3 \times 4 \mathrm{~mm}$ (fig. 2).

They were manufactured at a scale of $1: 1$ and at a scale of 1: 2, in six male-female (M-F) typo dimensions: circle, eight, cleat, triangle, wave and square.
From the point of view of their functionality we distinguish closed structures, which take all degrees of freedom from the plane (circle, eight, triangle and square) and open structures, which allows the axial displacement of the components (cleat and wave). 


\section{The Manufacturing Technology}

The all micro structures were performed by laser lithography, using SU8 resist, on 2 inches silicon wafers, and on polished brass wafers of 4 inches.

The plates were degreased in isopropyl alcohol, pickled in 6\% hydrochloric acid, washed with deionized water and dried with compressed air.

The SU8 3050 photoresist was deposited by centrifugation on a Spin Coating machine APT POLOS type.

Wafers fixing on the machine was done with vacuum and the working regime was: $30 \mathrm{~s}$ at 500 $\mathrm{rpm}, 60 \mathrm{~s}$ at $1000 \mathrm{rpm}$ and $45 \mathrm{~s}$ at $500 \mathrm{rpm}$.

The dose of SU 8 recommended by the manufacturer (MICRO CHEM) of $2 \mathrm{ml}$ has been observed. For a better adhesion of the photoresist to the wafers, before depositing the photoresist, a primer MCC 80/20 type, produced by MICRO CHEM, was also deposited.

A uniform photoresist layer of $101 \mu \mathrm{m}$ thick was obtained. The layer was measured using a Renishaw measuring system of a KernMicro type five-axis machining center. For thermal stabilization and removing the internal stress of the SU8 layer, a baking was done for 30 minutes at $95^{\circ} \mathrm{C}$.

The achievement of the micro structures implied the following operations [9], [10]:

a) Achieving the desired part drawing in a format accepted by the laser photolithography system DWL66 FS (DXF, CIF, Gerber, GDSII), in accordance with the shapes from figure 2 ;

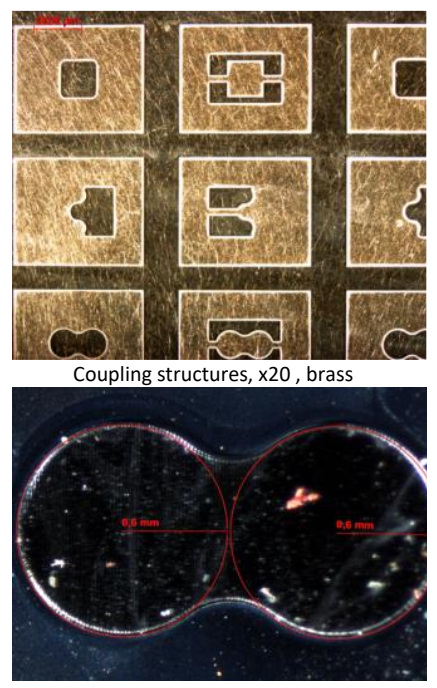

Eight form structure, male, $x 40$, silicon

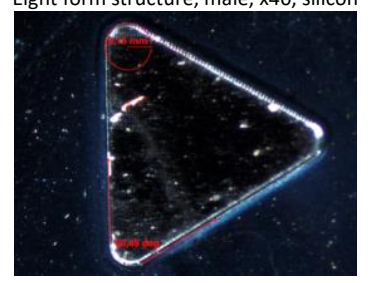

Triangle structure, male, $x 40$, silicon b) Converting the drawing into LIC format (matrix of points) and positioning each part on the support (MAP file), in a matrix format;

c) Setting the exposure parameters (making the JOB file);

d) The exposure using the laser lithography system, DWL66 FS;

e) The post-exposure baking; setting of the two value for the time and temperature [11];

The development of the unexposed SU8 photoresist was achieved by immersion in mr-Dev 600 solvent type. They have been made experiments, ranging the immersion time of the microstructure in the solvent, but also experiments with intercalation of rinses in 2-propanol (ICAO: isopropanol), to remove the resist from the small-sized channels. The developing temperature was the room temperature $\left(21-25^{\circ} \mathrm{C}\right)$, and there were made developments without shaking, with vigorous stirring and intermediate variants.

The developing were made as soon as the sample reached room temperature, and also after the expiry of a time to relax the structures.

The final rinse was done with 2-propanol and the drying was realized with compressed air or natural convection.

\section{Practical Results}

Conclusive structure images after the optimization of exposure and development technological process are presented in figure 3 . The micro structure images were made with a stereo optical microscope type Stemi 2000-C, by Zeiss.
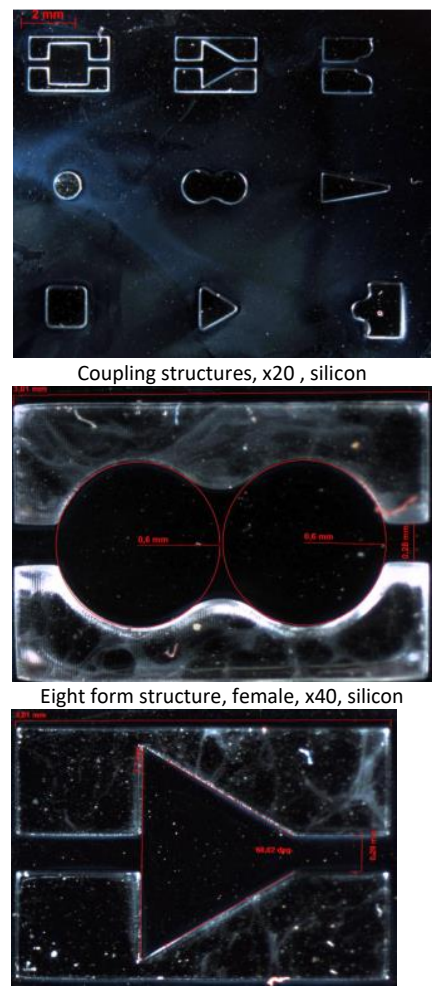

Triangle structure, male, $\times 40$, silicon 


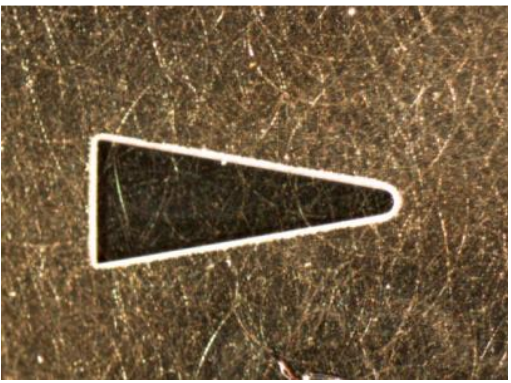

Cleat structure, male, $x 40$, brass

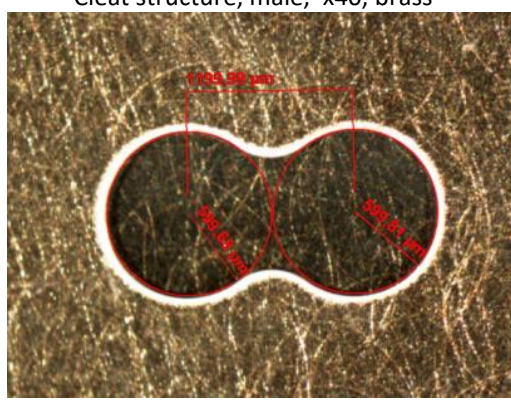

Eight form structure, male, $x 40$, silicon

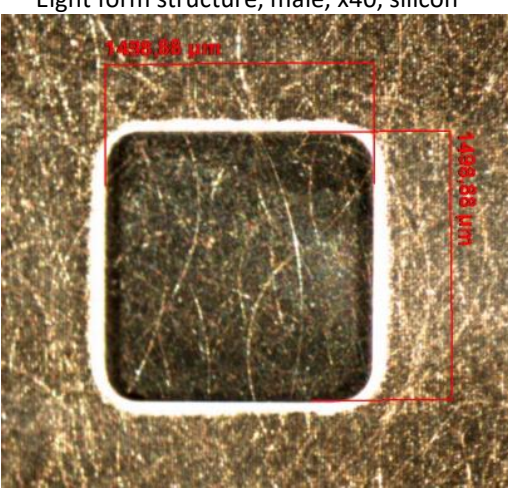

Square form structure, male, $x 40$, brass

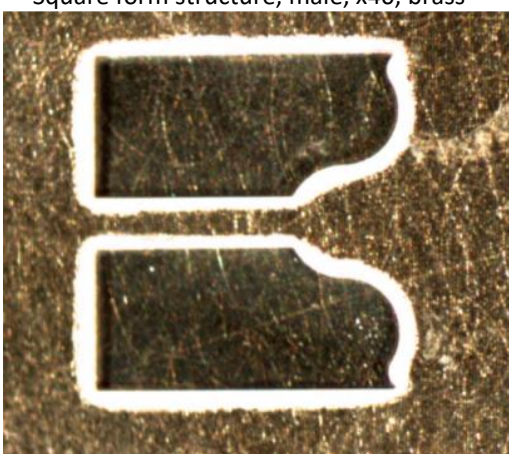

Wave form structure, female, $x 40$, bras

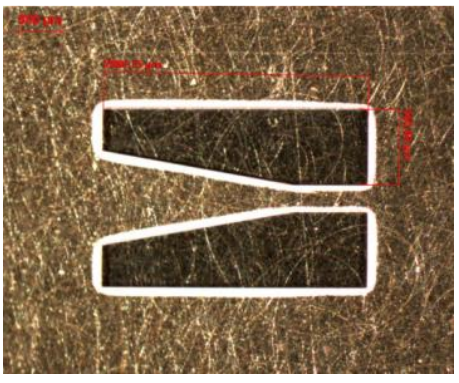

Cleat structure, female, $x 40$, brass

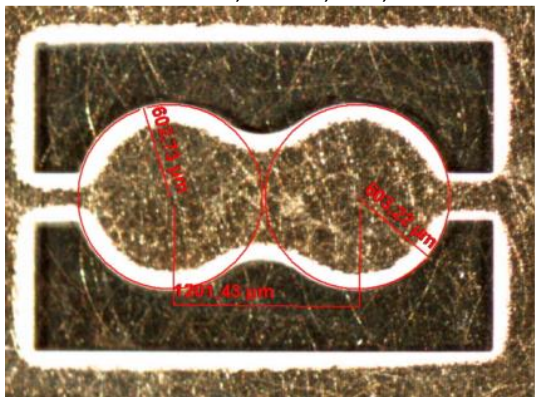

Eight form structure, female, $x 40$, brass

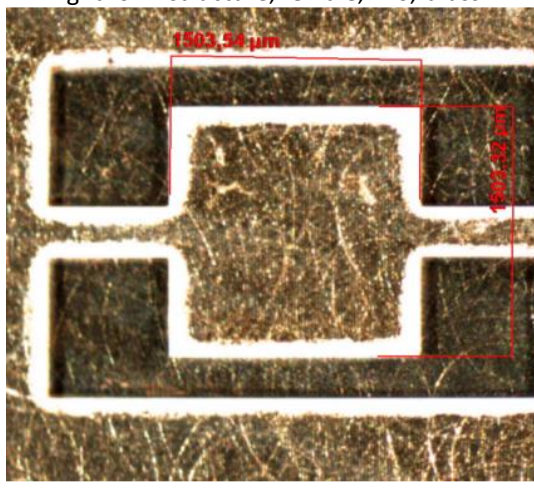

Square form structure, female, $\times 40$, brass

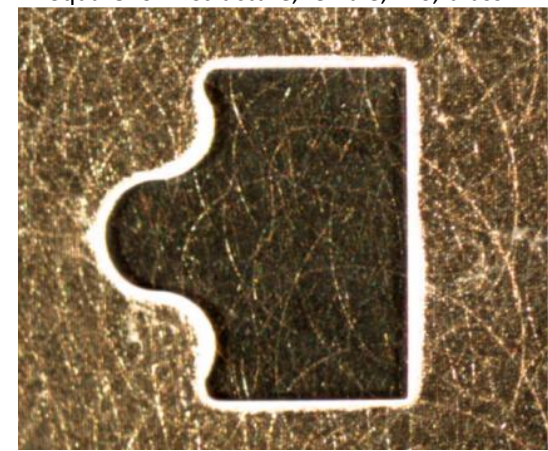

Wave form structure, male, $x 40$, brass

Fig. 3. Male-female coupling structures- optical microscopy

Figure 3 shows correctly processed male-female coupling structures, with dimensions embedded in the processing tolerances.

\section{The Measurement of the Coupling Structures}

The measurements were made using optical microscopy and white -light interferometry (optical profilometry).
Has been used a Wyko NT1100, which is a highprecision optical microscope profile with a Mirau interferometer and that can view areas with a resolution of up to $0.2 \mathrm{~nm}$ on the $\mathrm{Oz}$ axis using the interference fringe technique.

Dimensional measurements were performed for the coupled parts male-female type, also, the surface roughness was measured and the thickness uniformity of the structures.

In figure 4 it can be seen measured images for several analyzed coupling structures 


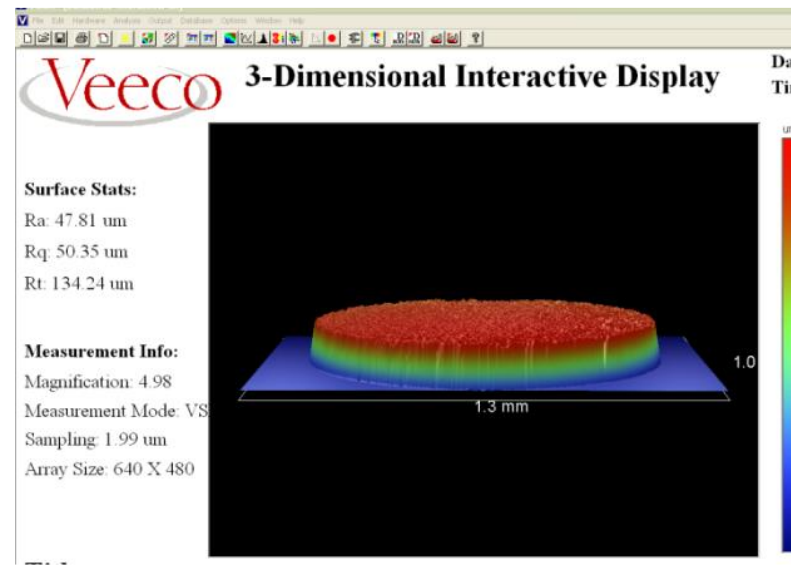

The 3D shape of the male circle structure

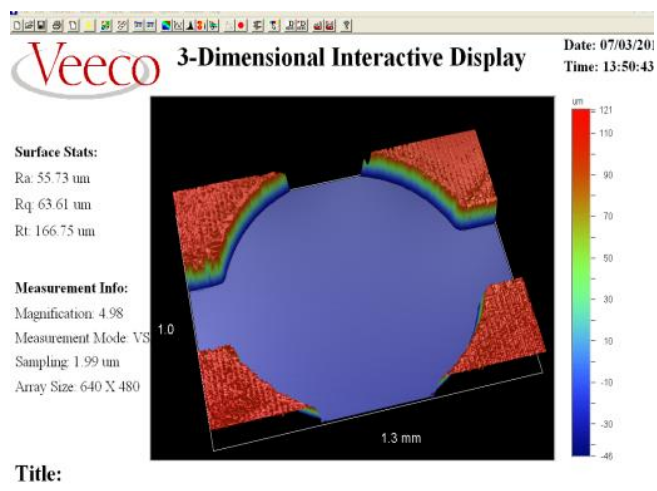

The 3D shape of the female circle structure

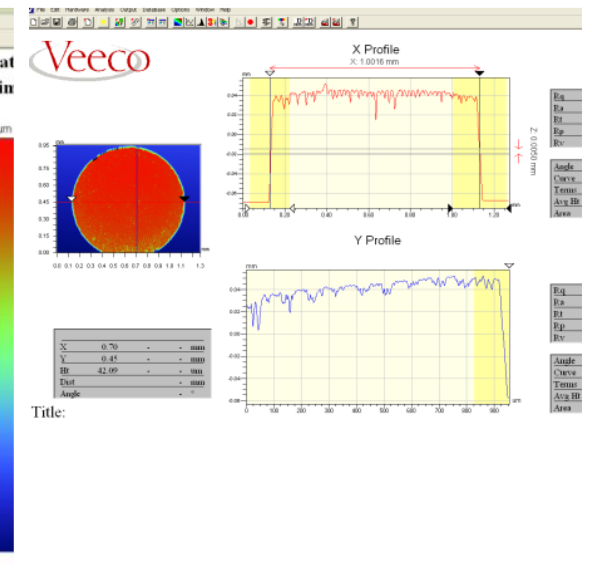

Measuring of the diameter to the male structure
Fig. 4. Measurements of coupling structures - white -light interferometry

Figure 4 shows us the 3D measurements of the malefemale structures. A good dimensional correlation is observed, the gap between those structures being less than $0.2 \mu \mathrm{m}$.

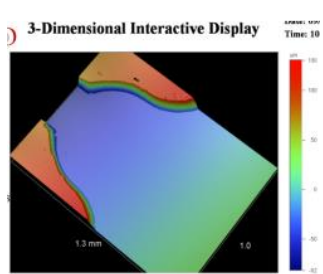

WAWE F

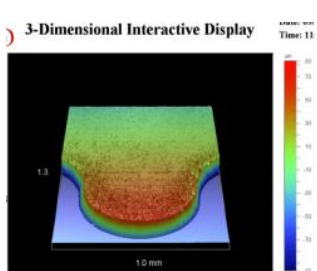

WAVE M

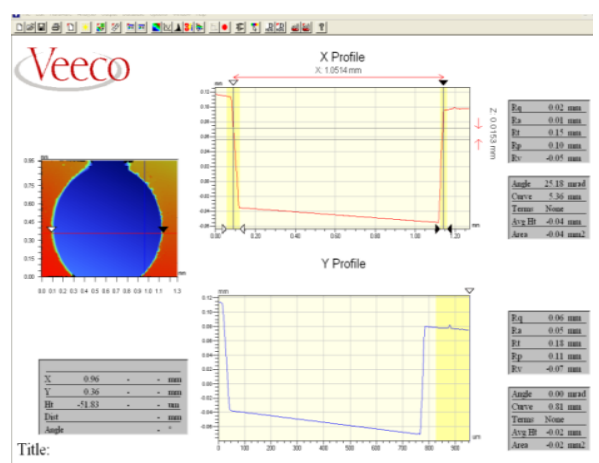

Measuring of the diameter to the female structure
Figure 5 shows us the 3D measurements of the wave and triangle male-female structures.

After the measurements, they also proved to be in the same precision class, less than $1 \mu \mathrm{m}$.

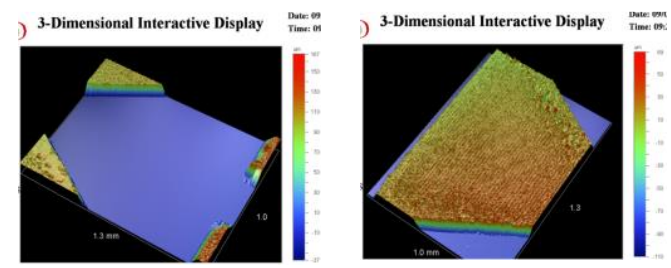

TRIANGLE M

Fig. 5. Male-female coupling structures - white -light interferometry

\section{Acknowledgements}

We acknowledge the Ministry of Research and Innovation (MCI), who made this research possible by financing the contract $249 / 2014$, with the title Electromagnetic and electrodynamic actuators manufactured through LIGA technology. We also thank the Polytechnic University of Bucharest who led this research and the National Institute for Research and Development in Electrical Engineering ICPE-CA from Bucharest, in whose laboratories the analyzed structures were processed and measured.

\section{Conclusions}

The conclusions establish the best choice of the constructive shapes for the connecting elements, according to a particular application.

All structures analyzed were dimensionally correct, with minimal gap of less than $1 \mu \mathrm{m}$.

For our application - electromagnetic microactuators - was preferred use of closed structures that ensure both accurate positioning and fixing of the two elements. 
From the constructive point of view, the most suitable form is the circle type, which allows the manufacture of the female structure also by a much simpler method, the drilling.

The results allow us to built a physical model of the electromagnetic microactuator and confirm the feasibility of the proposed idea.

This connection-fixing system can be extended to a large range of applications in the microstructure field, the problem of assembling microstructures is still of great interest in the world.

\section{References}

[1] Cho, H. J.: Micromachined permanent magnets and their mems applications, Doctorate of Philosophy (ph.d.), University of Cincinnati (2002).

[2] Judy, J.W., Muller, R.S.: Sensors Actuators (Physical A) A 53, 392 (1996).

[3] Dutoit, B. M., et.al.: High Performance Micromachined Sm2Co17 Polymer Bonded Magnets, Sensors and Actuators 77, pp. 178-182 (1999).
[4] Ahn, C.H., Allen, M.G., IEEE Trans. Ind. Electron. 45, 866(1998).

[5] Chin, T.: Permanent Magnet Films for Applications in Micromechanical Systems, J. Magn. Magn. Mater, 209, pp. 75-79 (2000).

[6] Guckel, H.: Progress in Magnetic Microactuators, Mycrosystem Technologies, 5, pp. 59-61 (1998).

[7] Tanase, N. , Morega, M., Comeaga, D., Ilie, C.: Bending Mode Cantilver Actuators forMicroElectromechanical Systems", Advanced Topics in Electrical Engineering (ATEE), 9th International Symposium on pag. 556 - 561 (2015).

[8] Demian, T., Tudor, D., Grecu, E.: Mecanisme de Mecanica Fina, EDP, Bucuresti, (1982).

[9] Ilie, C., Popa, M., Prioteasa, P., Chirita, I., Tănase, N.: Application of LIGA Technology for the Development of Micromechanical Systems, UPB Scientific Bulletin, Vol 73, Iss 2, p. 137-150 (2011).

[10] Ilie, C., Comeaga, D., Dontu , O., Popa, M.: Micro Parts Errors to Precision Manufacturing Using UV-LIGA, Technology, Advanced Materials Research Vols. 816-817 pp 237-241 (C) (2013).

[11] Prioteasa, P., Ilie, C., Popa, M., Iordoc, M., Sbarcea, B.G.: Electrodeposition of Nickel for Fabrication of Microfluidic Pumps, REVISTA de CHIMIE (Bucharest) Vol. 64 , No. 3, p. 275-280 (2013). 